\title{
Special issue on "Molecular genetics of aging and longevity": a critical time in the field of geroscience
}

\author{
Bérénice A. Benayoun ${ }^{1,2,3,4} \cdot$ Reiner A. Veitia ${ }^{5}$
}

Published online: 12 February 2020

(c) Springer-Verlag GmbH Germany, part of Springer Nature 2020

\begin{abstract}
Although aging was long considered as a simple and unavoidable decay, accumulating work over the last three decades have revealed that the process is not only highly regulated, but also dependent on external environmental inputs (Kennedy et al. 2014; Lopez-Otin et al. 2013). Indeed, the existence of a genetic basis underlying aging became evident in the early 1990s with the discovery of specific genetic mutations that could double the lifespan of the nematode C. elegans (Kenyon et al. 1993). Since this seminal discovery, a number of core "hallmarks" of the aging process have been described, including genomic instability, inflammation, mitochondrial dysfunction, loss of protein homeostasis (i.e., "proteostasis") and (epi-)genomic alterations (Kennedy et al. 2014; Lopez-Otin et al. 2013). Aging is the single greatest risk factor for many chronic diseases (e.g., Alzheimer's, cancer, cardiovascular dysfunction, diabetes, etc.), which have a growing medical, financial, and societal burden due to the growing aging population. Not surprisingly, clinical frailty involves a cumulative decline in anatomical, physiological and molecular functions and is apparent at multiple levels of biological organization: genome, epigenome, tissues, organs, and the organism. Indeed, the "geroscience hypothesis" posits that aging is the underlying cause leading
\end{abstract}

Bérénice A. Benayoun

bbenayou@usc.edu

Reiner A. Veitia

Reiner.VEITIA@ijm.fr

1 Leonard Davis School of Gerontology, University of Southern California, Los Angeles, CA 90089, USA

2 Molecular and Computational Biology Department, Dornsife College of Letters, Arts and Sciences, University of Southern California, Los Angeles, CA 90089, USA

3 USC Norris Comprehensive Cancer Center, Epigenetics and Gene Regulation, Los Angeles, CA 90089, USA

4 USC Stem Cell Initiative, Los Angeles, CA 90089, USA

5 Institut Jacques Monod, CNRS UMR7592, Université de Paris, Paris, France to the development of many chronic diseases (Kennedy et al. 2014), and thus requires an integrated research approach to human health. Detailed genome wide and populational investigations on the origins and effects of germline and somatic genetic and epigenetic alterations in interaction with the environment will open up the possibility to fully understand the process(es) of aging. From the perspective of precision medicine, it will be possible to identify individuals at higher risk of developing single or multiple morbidities for devising new preventive measures, such as specific senolytic treatments.

In this Special Issue, we assembled a collection of articles from expert in various aspects of aging biology, aimed to both lay out novel advances in the characterization of aging hallmarks (i.e., protein homeostasis, stem cell decline, genomic instability, and chromatin alterations), as well as highlight emerging topics of interest in the field of aging biology (i.e., importance of sex differences, novel aspects of genomic dysregulation such as miRNA or splicing, and mitochondrial genomics). The first conceptual set of articles spans topics that summarize and expand our knowledge of existing hallmarks of aging (Grassmann et al. 2019; Innan et al. 2019; McNeely et al. 2019; Wong et al. 2019; Yu et al. 2020). In the review by Wong and colleagues, the impact of autophagy on aging and longevity, a key mechanism in recycling cellular macromolecules which is key for proteostasis, is explored (Wong et al. 2019). The review by McNeely and colleagues explores the impact of lifelong DNA-damage accumulation of long-lived stem cells (McNeely et al. 2019), whereas the review by Yu et al. (2020) highlights loss of chromatin integrity as an important source of genotoxic stress with aging. The original study by Grassman and collaborators explores progressive Y-chromosome aneuploidy in patients with macular degeneration (Grassmann et al. 2019). Innan and colleagues propose a demographic model to explain age-related frailty and morbidity through mutation and epimutation accumulation (Innan et al. 2019). 
A second conceptual set of articles in this Issue cover emerging topics in aging research (Bhadra et al. 2019; Kinser and Pincus 2019; Reynolds et al. 2020; Sampathkumar et al. 2019). The review by Bhadra and colleagues highlights loss of precision in alternative splicing as emerging features of aging (Bhadra et al. 2019). In their review, Kinser and Pincus provide insights into the role of non-coding miRNA as master molecular regulators of the aging process, as well as biomarkers of aging (Kinser and Pincus 2019). Reynolds et al. discuss the intriguing impact of the breakdown of communication between the mitochondria and nuclear compartments during aging, and the importance of considering both of our genomes (i.e., nuclear and mitochondrial) in the study of aging genomics (Reynolds et al. 2020). Finally, Sampathkumar and colleagues summarize and discuss the overwhelming presence of sex-dimorphic phenotypes in aging, longevity, and age-related diseases and highlight the importance of considering sex as a point of interest in the study of aging rather than an incovenience to be taken into account (Sampathkumar et al. 2019).

We are grateful to the all the authors who have contributed cutting-edge scholarly work, and we thank the Human Genetics editorial and production staff for assisting us in realizing our vision in this overview of current hot questions in the aging biology field.

Funding Bérénice A. Benayoun is supported by NIA R00AG049934 and R21AG063739, an innovator grant from the Rose Hills foundation, a seed grant from the NAVIGAGE foundation, and a generous gift from the Hanson-Thorell Family.

\section{References}

Bhadra M, Howell P, Dutta S, Heintz C, Mair WB (2019) Alternative splicing in aging and longevity. Hum Genet. https://doi. org/10.1007/s00439-019-02094-6
Grassmann F, Weber BHF, Veitia RA, International AMDGC (2019) Insights into the loss of the $\mathrm{Y}$ chromosome with age in control individuals and in patients with age-related macular degeneration using genotyping microarray data. Hum Genet. https://doi. org/10.1007/s00439-019-02029-1

Innan H, Veitia R, Govindaraju DR (2019) Genetic and epigenetic Muller's ratchet as a mechanism of frailty and morbidity during aging: a demographic genetic model. Hum Genet. https://doi. org/10.1007/s00439-019-02067-9

Kennedy BK, Berger SL, Brunet A, Campisi J, Cuervo AM, Epel ES, Franceschi C, Lithgow GJ, Morimoto RI, Pessin JE, Rando TA, Richardson A, Schadt EE, Wyss-Coray T, Sierra F (2014) Geroscience: linking aging to chronic disease. Cell 159:709-713. https ://doi.org/10.1016/j.cell.2014.10.039

Kenyon C, Chang J, Gensch E, Rudner A, Tabtiang R (1993) A C. elegans mutant that lives twice as long as wild type. Nature 366:461-464. https://doi.org/10.1038/366461a0

Kinser HE, Pincus Z (2019) MicroRNAs as modulators of longevity and the aging process. Hum Genet. https://doi.org/10.1007/s0043 9-019-02046-0

Lopez-Otin C, Blasco MA, Partridge L, Serrano M, Kroemer G (2013) The hallmarks of aging. Cell 153:1194-1217. https://doi. org/10.1016/j.cell.2013.05.039

McNeely T, Leone M, Yanai H, Beerman I (2019) DNA damage in aging, the stem cell perspective. Hum Genet. https://doi. org/10.1007/s00439-019-02047-z

Reynolds JC, Bwiza CP, Lee C (2020) Mitonuclear genomics and aging. Hum Genet. https://doi.org/10.1007/s00439-020-02119-5

Sampathkumar NK, Bravo JI, Chen Y, Danthi PS, Donahue EK, Lai RW, Lu R, Randall LT, Vinson N, Benayoun BA (2019) Widespread sex dimorphism in aging and age-related diseases. Hum Genet. https://doi.org/10.1007/s00439-019-02082-w

Wong SQ, Kumar AV, Mills J, Lapierre LR (2019) Autophagy in aging and longevity. Hum Genet. https://doi.org/10.1007/s00439-01902031-7

Yu R, McCauley B, Dang W (2020) Loss of chromatin structural integrity is a source of stress during aging. Hum Genet. https://doi. org/10.1007/s00439-019-02100-x

Publisher's Note Springer Nature remains neutral with regard to jurisdictional claims in published maps and institutional affiliations. 